\title{
Ergebnisse: Während des Studiums
}

\subsection{Sprachkurs in Deutschland}

Wie gerade geschildert, müssen, bevor ein Studium in Deutschland aufgenommen werden darf, Grundkenntnisse in der deutschen Sprache nachgewiesen werden. Generell werden Deutschkenntnisse auf dem Sprachniveau von B2 verlangt. Deswegen bietet der KAAD seinen Stipendiat*innen einen Deutschkurs in Bonn an, den die Stipendiat*innen vor ihrem Studium absolvieren können. Je nach bereits vorhandenen Deutschkenntnissen kann der Sprachkurs zwischen einem Monat und sechs Monaten dauern. Die kompletten Kosten dafür übernimmt der KAAD. Diejenigen Stipendiat ${ }^{*}$ innen, die bereits schon vorher einen Deutschkurs entweder in Deutschland - falls sie zum Zeitpunkt der Bewerbung schon in Deutschland waren - oder im Herkunftsland erfolgreich absolviert haben oder die deutsche Sprache bereits schon in der Schule oder privat gelernt haben (s. o.), müssen den Deutschkurs des KAAD in Bonn nicht belegen. Allerdings haben nur die wenigsten der Befragten einen Deutschkurs im Herkunftsland vorher belegt. Ein unserer Untersuchung betraf dies nur ein paar wenige Alumni. Der KAAD kooperiert dabei mit dem Kreuzberg-Institut in Bonn und schickt seine Stipendiat*innen zum Deutschkurs regelmäßig dorthin. Alle anfallenden Kosten (Unterricht, Übernachtung und Verpflegung) werden dabei vom KAAD übernommen. Je nach Vorkenntnissen und sprachlichen Anforderungen im späteren Studium dauert der Sprachkurs zwischen vier und sechs Monaten.

Der gemeinsame Sprachkurs hilft vielen dabei, die Probleme zu Beginn ihrer Zeit in Deutschland leichter zu bewältigen. Das liegt vor allem daran, dass die Stipendiat*innen während dieser Zeit in der Regel zusammen in einem Wohnheim in Bonn wohnen und sich bei Problemen wie Heimweh oder dem Erlernen der deutschen Sprache gegenseitig unterstützen können. Überhaupt haben die meisten Alumni, die einen Sprachkurs in Bonn belegt haben, sehr positive Erinnerungen an diese Zeit:

S. Krannich und U. Hunger, Studierendenmigration und Entwicklung, https://doi.org/10.1007/978-3-658-32048-5_4 
"Ich habe den Deutschkurs des KAAD in Bonn im Frühling und Sommer 2000 besucht. Der hat mir sehr gut gefallen und hat mir über die schwere Anfangszeit in Deutschland hinweggeholfen, weil viele gleichaltrige andere Stipendiaten des KAAD mit ähnlichen Erfahrungen aus verschiedenen Ländern mit mir den Kurs zusammen gemacht haben. Wir konnten uns gegenseitig über die schwierige Anfangszeit helfen und haben zusammen die Hausaufgaben für den Deutschkurs gemacht" (Interview mit einem ghanaischen Alumnus in Kumasi, Ghana, 2018).

„Das war eine sehr schöne Zeit für mich, weil ich da viel Zeit hatte, wohnte mit anderen Stipendiaten aus Afrika zusammen, mit denen ich viel zusammen unternommen habe, und Deutsch dabei gelernt habe" (Interview mit einer ghanaischen Stipendiatin, Skype, 2017).

„Wir haben auch viel zusammen gemacht, zusammen gekocht, ins Café gegangen und Ausflüge gemacht. Dabei konnten wir uns immer zusammen über die komplizierte deutsche Sprache aufregen" (Interview mit einer ghanaischen Stipendiatin in Bonn, 2017).

Nach der Zeit des gemeinsamen Sprachkurses in Bonn, verteilen sich die Stipendiat ${ }^{*}$ innen an die einzelnen Universitäten in ganz Deutschland. Häufig fühlen sie sich dort zunächst wieder einsamer, weil viele der während des Sprachkurses neu gewonnenen Freund ${ }^{\star}$ innen dann wieder fehlen. Deswegen ist auch die KAADJahresakademie immer wieder ein freudiger Anlass zum Feiern für viele, weil sie dort ihre Freund ${ }^{*}$ innen aus den gemeinsamen Sprachkursen wiedersehen. Bonn ist deshalb eine positive Symbolstadt für viele Stipendiat*innen.

Es gibt auch Alumni, die nach ihrem Vorbereitungssprachkurs noch einen weiteren Sprachkurs während des Studiums besuchen, wenn die Deutschkenntnisse noch nicht ausreichend für das Studium waren.

"In Germany, with the KAAD at the Kreuzberg Institute in Bonn: When you get the scholarship, you get this famous course. For me, it was three or four months before I could start my Master. For me my first semester was the most horrible thing ever, because I could not understand any of the things they were talking about, because it was a really specific kind of language, and I had only four months of German. So, it was only the basic stuff, just to speak on the street or go to the grocery store, but not for specialized classes. So, for me it was the most difficult time, more difficult than writing my Master's thesis. It was crazy, my mind was full with the notes and words, and grammar and 
everything possible. To write in German was very difficult, and my defense was also in German. [...] I could get only these four months. I think it was because of the agreement with my university in Colombia. Because normally the KAAD gave like up to one year German class or something like that. I got only four months, and the rest was doing part of my first semester. I was taking some courses that the KAAD was also funding at the Goethe Institute in Dresden, and I was preparing myself for the test. [...] Yes, because I had to continue with my German classes, because I was asking the KAAD for more funding for my German classes, because I was not able to understand everything in my classes at the TU Dresden. And the KAAD gave me this course at the Goethe Institute in Dresden." (Interview mit einer kolumbianischen Alumna in Deutschland, Skype, 2017).

Diejenigen, die bereits in Deutschland waren, als sie sich für ein KAAD-Stipendium beworben haben (S2-Stipendienprogramm), haben ebenfalls oftmals vor ihrem Studium noch einen Deutschkurs belegt, wie z. B. ein indonesischer Alumnus, der einen Deutschkurs am Studienkolleg belegt hat, während er dort gleichzeitig seine Hochschulreife für ein Studium an einer deutschen Hochschule erworben hat. Eine andere indonesische Alumna belegte zuerst für ein komplettes Jahr einen Intensiv-Deutschkurs in Berlin, bevor sie dort ihr Studium an der Technischen Universität aufnahm. Eine ghanaische Alumna entschied sich dafür, nur einen einmonatigen Sprachkurs an der Universität Bonn zu machen, weil sie ihre spätere Doktorarbeit an der Universität auf Englisch verfasste und auch mit den Kolleginnen nur auf Englisch kommunizierte. Während ihrer gesamten fast fünfjährigen Zeit in Deutschland lernte sie nur ein paar Wörter Deutsch. Ein kolumbianischer Alumnus machte dagegen einen achtmonatigen Deutschkurs an der Universität Gießen bevor er dort mit seinem Studium anfing. Ein anderer kolumbianischer Alumnus absolvierte sowohl einen Deutschkurs am Goethe Institut in Bogotá für eineinhalb Jahre als auch später in Marburg, bevor er sein Jurastudium dort anfing. Er wollte noch einen zweiten Deutschkurs belegen, weil er sich nach dem ersten noch nicht gut auf sein Jurastudium vorbereitet sah:

„Ja, aber das hat doch nicht gereicht. Ich brauchte noch ein halbes Jahr in Marburg. Ich habe dort sehr, sehr fleißig Deutsch gelernt. Und nachdem ich den Kurs beendet hatte, habe ich in der Juristischen Fakultät angefangen, um die juristische Sprache zu beherrschen. [...] Zu allererst, die deutsche Sprache zu beherrschen. Mir war klar, dass wenn ich den Sprachkurs am Anfang nicht bestehen würde, dann müsste ich nach sofort nach Kolumbien zurück. Deswegen habe ich am Anfang sehr hart Deutsch gelernt. Dann natürlich das tägliche Leben. Am Anfang war ich in Deutschland absolut alleine. Das war nicht so einfach, aber immer mit Lust 
und Liebe kann man alles. Deswegen habe ich das mit großer Freude gemacht, und das war gut" (Interview mit einem kolumbianischen Alumnus in Bogotá, Kolumbien, 2017).

\subsection{Studium in Deutschland}

Nach dem KAAD-geförderten Sprachkurs in Bonn und/oder eventueller anderer Sprachkurse fangen die Stipendiat*innen in der Regel direkt mit dem Studium oder der Promotionsarbeit an einer deutschen Universität an. In unserer OnlineBefragung waren über die Hälfte der Alumni für ein bis drei Jahre für ein Studium oder einen Forschungsaufenthalt in Deutschland (50,11\%, 223 von 445 befragten Alumni). Fast ein Fünftel (84 bzw. 18,88 \%) gab sogar an, für bis zu sechs Jahren für ein Studium oder einen Forschungsaufenthalt in Deutschland gewesen zu sein, und weitere fünf Prozent für sieben bis neun Jahre (22 bzw. 4,94\%). Von den Befragten hatten zudem fast alle bereits ein Studium im Herkunftsland absolviert. Die meisten der Befragten (über drei Viertel, 344 von 354 bzw. 77,3\%) waren bisher einmal mit einem KAAD-Stipendium für ein Studium oder einen Forschungsaufenthalt in Deutschland, knapp 12 Prozent (53 bzw. 11,91\%) zweimal und weitere rund zehn Prozent sogar noch häufiger.

Die Stipendiat*innen wählten zudem häufig Themen für ihre Abschluss- oder Doktorarbeiten aus, die auch relevant waren für die Entwicklung des Herkunftslandes. So haben sich Georgier*innen in ihren Abschluss- und Promotionsarbeiten hauptsächlich mit Rechtsfragen in Bezug auf das georgische Rechtssystem beschäftigt. Interessanterweise sind das vor allem junge Alumni zwischen 25 und 35 Jahren, die erst in den letzten fünf Jahren in Deutschland für einen Master- oder Promotionsaufenthalt waren, aber bereits mehrjährige Berufserfahrung im georgischen Staats- oder Rechtswesen oder als Dozent ${ }^{\star}$ innen an georgischen Universitäten vor ihrem Studienaufenthalt in Deutschland gesammelt haben. Dabei beschäftigen sie sich in ihren Master- und Doktorarbeiten immer mit einem spezifischen Rechtsvergleich zwischen dem deutschen und georgischen System. So befasst sich z. B. ein Alumnus in seiner Doktorarbeit mit Naturrechtsphilosophie und Grundrechten in Deutschland und Georgien im Vergleich. Ein anderer Alumnus untersucht in seiner Arbeit die Unterschiede im deutschen und georgischen Strafrecht und ein weiterer Alumnus die Unterschiede speziell im Jugendstrafrecht.

Mit dem Einfluss des deutschen Handelsrechts auf das georgische Handelsrecht im Rahmen der Freihandelsabkommen zwischen der EU und Georgien beschäftigte sich eine Alumna in ihrer Masterarbeit an der Humboldt-Universität in Berlin. Sie 
bekam ein sechsmonatiges Stipendium vom KAAD. Für ihr Studium in Deutschland unterbrach sie für ein Jahr ihre Tätigkeit als Rechtsberaterin an der oben genannten Nationalagentur für das staatliche Eigentum (AdöR). Ein weiterer Alumnus absolvierte einen KAAD geförderten Forschungsaufenthalt im Rahmen seiner Doktorarbeit zum Thema „Strafrecht für Minderjährige. Ein Vergleich zwischen Deutschland und Georgien" an der Ruhr-Universität in Bochum. Neben seinem Studium engagierte er sich in der NGO „Georgian Young Lawyers Association“, die sich für die Umsetzung demokratischer Rechte und Werte in Georgien einsetzt. Er plant seine Zukunft als Rechtsanwalt in Georgien. Ein anderer Alumnus, der heute Juraprofessor an der East European University in Tiflis ist, forschte zum Thema „Perspektiven zur Implementierung eines Zweikammernsystems in Georgien“ am Max-Planck-Institut für Ausländisches und Öffentliches Recht in Heidelberg.

Die palästinensischen Stipendiat*innen haben sich im Rahmen ihres Studiums und ihrer Promotion vornehmlich mit medizinischen und gesundheitsrelevanten Themen auseinandergesetzt. Themen der Forschungsarbeiten sind zum Beispiel „Neue therapeutische Ansätze in der Behandlung von Frühgeborenen“ in den 1990er Jahren an der Ludwig-Maximilians-Universität (Promotion). Ein anderes Beispiel ist ein Alumnus, der Innere Medizin mit Spezialisierung auf Diabeteskrankheiten von 1999 bis 2006 an der Universität des Saarlandes in Saarbrücken studiert hat. Viele machten zudem eine Facharztausbildung in Deutschland. Andere promovierten mit Unterstützung eines KAAD-Stipendiums im Fachbereich Psychologie, mit Schwerpunkten in der klinischen sowie Arbeits- und Organisationspsychologie. Themen der Doktorarbeiten waren etwa „Phonologische Prozesse bei arabischsprechenden Kindern mit einer funktionellen Dyslalie“, „Effectivity of Multiple Context Exposure in Attenuating Return of Fear and the Mechanism behind it" (am Beispiel palästinensischer Bewohner*innen in der West Bank). Zudem gibt es Masterarbeiten zum Thema „The Prevalence and Determinants of Post-Traumatic Stress Disorder (PTSD) among Palestinian Children Exposed to Political Violence: a Systematic Review and Meta-Analysis“ oder die Diplomarbeit zum Thema „Entwicklungsverläufe und Entwicklungsmerkmale in den Zeichnungen palästinensischer Kinder“. Andere Arbeitsthemen mit Bezug auf Palästina waren „Assiwar - Palestinian Feminist Movement in Support of Victims of Sexual Abuse“, „Islam und sozialer Wandel zwischen politischem Realismus und religiöser Pflicht am Beispiel der Muslimbrüder in Jordanien“ und die „Analyse der Denkmalpflege in den palästinensischen Autonomiegebieten“.

Ghanaische Stipendiat*innen setzen sich in ihren Master- oder Forschungsarbeiten überwiegend mit landwirtschaftlichen Themen in Ghana auseinander, und zwar disziplinübergreifend, d. h. unabhängig ihrer Forschungsdisziplin. So schrieb z.B. eine Alumna, die Geoinformatik in Stuttgart studiert hat und heute für ein 
deutsches Landvermessungsunternehmen arbeitet, ihre Masterarbeit zum Thema „Estimation of Forest Biomass in Ghana". Ein anderer Alumnus schrieb seine wirtschaftswissenschaftliche Doktorarbeit über „Agricultural Export in Ghana and its Connections to Local Supply Development". In diesem Bereich forscht er weiterhin. Eine andere Alumna, die heute Landwirtschaft an der Universität Legon in Accra lehrt, schrieb ihre Doktorarbeit über das Thema „Marketing and Market Queens - A Study of Tomatoe Female Farmers in the Upper East Region of Ghana“. Dabei untersuchte sie die wirtschaftlichen Herausforderungen für Kleinbäuerinnen in der Tomatenproduktion in Nordghana. Ein Alumnus, der in Bonn zum Thema „Import Liberalization of Agricultural Commodities: Implications for Food Security, Rural Income and Poverty in Sub-Sahara Africa" forschte, untersuchte die Zusammenhänge zwischen Marktliberalisierung und Nahrungsmittelverteilung in Nordghana. Ähnliche Themen wurden auch in den Arbeiten „Global Food and Global Security“, „Emergent Urban Land Market and Inter-Generational Land Relations in North Western Ghana“, „Innovation Management in Knowledge Intensive Business Services in Ghana", „Urban Growth in Ghana: The Socio-Economic and Environmental Implications in the Wa Municipality" behandelt. Dabei geht es immer um die zentrale Frage, inwieweit marktwirtschaftliche Strukturen die Lebensmittelversorgung in Ghana verbessern können und inwieweit staatliche Behörden dabei korrigierend eingreifen sollten, um eine bessere Lebensmittelverteilung in Ghana sicherzustellen. Das ist insbesondere im Norden Ghanas ein zentrales Problem, da aufgrund von ungünstigen Umweltfaktoren (Trockenheit, unfruchtbare Böden etc.) und fehlender Infrastruktur eine gute Lebensmittelversorgung der Bevölkerung nur bedingt gewährleistet werden kann. Dies ist auch ein zentraler Grund für relativ überteuerte Lebensmittelprodukte in Ghana.

Andere Arbeiten der ghanaischen Stipendiat*innen und Alumni beschäftigen sich mit umweltpolitischen Aspekten und Umweltverschmutzung, wie z.B. „Woodfuel Consumption, Deforestation and Farmer Adoption of Agroforestry Practices for Woodfuel Production: A Case Study of the Ashanti Region of Ghana", "Mercury Concentrations in Biological, Water and Soil Samples from Artisanal Gold Mining Communities in Adansi Traditional Area“ oder "Arsenic Contamination of Groundwater in and around Obuasi Municipality of Ghana“. Allein am interdisziplinären Zentrum für Entwicklungsforschung (ZEF) der Universität Bonn haben sechs ghanaische KAAD-Alumni promoviert, die sich alle in ihrer Arbeit mit einem Thema aus dem Umwelt-, Energie- und Landwirtschaftsbereich in Ghana beschäftigt haben. Darunter war auch eine ghanaische Alumna, die im Rahmen des sog. Glowa-Forschungsprojektes, das vom ZEF durchgeführt wurde, promoviert. Das Glowa-Forschungsprojekt war ein großes Projekt, das sich mit der 
Entwicklung von nachhaltigen Bewässerungssystemen für die Landwirtschaft in Nordghana beschäftigt (Interview mit ghanaischen Stipendiaten, Ghana, 2018).

Ein aktueller Stipendiat, der im Bereich "geografische Entwicklungsforschung“ an der Universität Bayreuth promoviert, beschäftigt sich mit den Auswirkungen von Globalisierung und Klimawandel auf die gemeinschaftliche Landnutzung in Kommunen im Süden Ghanas. Im Rahmen seines Promotionsprojekts geht er jedes Jahr für ca. drei Monate nach Ghana, um Feldforschung in den ausgewählten Kommunen vor Ort zu machen. Er interviewt dann lokale Akteur ${ }^{*}$ innen und führt Beobachtungen in kommunalen Einrichtungen durch (Interview mit ghanaischen Stipendiaten, Bonn, 2017).

Kolumbianische Stipendiat ${ }^{\star}$ innen setzten sich demgegenüber in ihren Examensarbeiten vor allem mit dem Friedens- und Demokratieprozess in Kolumbien auseinander. Typische Titel von Master- und Doktorarbeiten sind „Die Entwicklung von demokratischen Strukturen in indigenen Kommunen Kolumbiens nach dem Rückzug der FARC-Guerillas“, „Die Rolle der Jugend bei der Entwicklung in der Post-Konfliktphase am Beispiel der Stadt Villavicencio und des Departements Meta“ und „Empowerment and Representativeness of Gender: Perspectives from the Violence and Crime in the Case of Bogotá, Colombia". Ein bereits oben erwähnter kolumbianischer Alumnus, der Internationales Strafrecht im LLM-Studiengang an der Universität Freiburg studierte, beschäftigte sich in seiner Masterarbeit mit der Rolle des Internationalen Strafgerichtshofs im Friedensprozess in Kolumbien. Er untersuchte die Frage, inwieweit der Internationale Strafgerichtshof für Verbrechen im Rahmen des Bürgerkriegs in Kolumbien zuständig ist, für den Fall, dass die kolumbianische Justiz Bürgerkriegsverbrechen nicht verfolgt.

Ein weiterer Alumnus studierte im Masterstudiengang „Intercultural Conflict Management" an der Alice Salomon Hochschule Berlin. Er schrieb seine Abschlussarbeit zum Thema „Rolle, Organisation und Repräsentanz von Frauen im Friedensprozess in Kolumbien". Er kam zu dem Ergebnis, dass Frauen am meisten unter dem Konflikt gelitten haben, aber im aktuellen Friedensprozess kaum eine Rolle spielen. Er schloss seine Masterarbeit im März 2017 ab (Interview mit einem kolumbianischen Alumnus in Deutschland, Skype, 2017). Darüber hinaus beschäftigten sich die kolumbianischen Alumni in ihren Arbeiten auch mit anderen Themen, die einen Bezug zum Herkunftsland Kolumbien oder einen Vergleich Kolumbiens mit Deutschland zum Inhalt hatten, wie z. B. die Doktorarbeit „Verwaltungsrecht im Vergleich: deutsches, französisches und kolumbianisches Recht" oder „Der Vergleich des Schriftstellers der deutschen Romantik Novalis mit den Schriften eines indigenen Schriftstellers aus dem Süden Kolumbiens“. Eine andere Alumna, die sich in ihrer MA-Arbeit mit Fortbildungsmöglichkeiten im Bereich „erneuerbare Energien“ für Grundschullehrer*innen in der Stadt Medellin in 
Kolumbien beschäftigte, erhielt den DAAD-Preis 2017 für ihre wissenschaftliche Leistung und ihr großes Engagement für die deutsch-kolumbianischen Hochschulbeziehungen (DAAD 2017).

Die Schwerpunkte der indonesischen Alumni liegen wiederum auf natur- und wirtschaftswissenschaftlichen Themen. In ihren Master- und Doktorarbeiten beschäftigten sie sich häufig mit Themen, die auch für Indonesien entwicklungsrelevant sind und auf die sie sich häufig in ihrem späteren Beruf in Deutschland oder Indonesien beziehen oder zurückgreifen konnten. Ein Alumnus, der von 1986 bis 1996 an der RWTH Aachen einen Diplom-Studiengang im Fach Elektrotechnik absolvierte, schrieb seine Diplomarbeit über das Thema „Glasfaseranwendung in der Produktion“. Der Alumnus entschloss sich damals bewusst, diesen Studiengang in Aachen zu studieren und über dieses Thema zu schreiben, weil die RWTH Aachen in diesem Bereich zu den führenden Forschungseinrichtungen weltweit gehört und man zu diesem Thema in Indonesien damals noch nicht forschen konnte. Nach seiner Rückkehr nach Indonesien, machte sich der Alumnus in Jakarta mit einem Telekommunikationsunternehmen selbstständig und konnte sich dabei auf sein im Studium erworbenes Fachwissen zu Elektrotechnik und Glasfaserherstellung stützen (Interview mit einem indonesischen Alumnus in Pontianak, Indonesien, 2017).

Ein anderer indonesischer Alumnus studierte 1998 und 1999 im Masterstudiengang "Global Production Engineering" an der TU Berlin und anschließend den Masterstudiengang Wirtschaftsingenieurwesen von 1999 bis 2002, ebenfalls an der TU Berlin. In diesen beiden Studiengängen schloss er sein Studium mit den Arbeiten zum Thema Handelsmöglichkeiten im Ingenieurbereich in Europa und Asien ab. Eine andere Alumna schrieb ihre Masterarbeit zum Thema „Private Owned Business Sector in Education“ im Rahmen des Studiengangs "Global Production Engineering" an der TU Berlin, den sie in den Jahren von 2000 bis 2003 absolvierte. Ihre Masterarbeit war entscheidend für die Idee, sich später in Indonesien in der Vermittlung von indonesischen Studenten an ausländische Universitäten selbstständig zu machen (Interview mit einer indonesischen Alumna in Jakarta, Indonesien, 2017).

Eine andere Alumna promovierte an der Ludwig-Maximilians-Universität in München im Bereich International Competition Law von 2006 bis 2011. Das Thema ihrer Arbeit war „Unilateral Restraints in the Retail Business: A Comparative Study on Competition Law in Germany and Indonesia“. Dabei verglich sie die Einzelhandelsbeschränkungen im Nahrungsmittelsektor zwischen Deutschland und Indonesien und untersuchte, was Indonesien von Deutschland übernehmen könnte. In ihrer Arbeit kam sie zu dem Ergebnis, dass Indonesien einige Einzelhandelsschutzbestimmungen von Deutschland übernehmen könnte, u. a. zum Schutz gegen Monopolbildung. Nach ihrer Promotion kehrte sie nach Indonesien zurück, 
um weiter als Rechtswissenschaftlerin an diesem Thema an der Partneruniversität des KAAD in Indonesien, der Atma Jaya-Universität, zu arbeiten (Interview mit einer indonesischen Alumna in Jakarta, Indonesien, 2017).

Eine aktuelle Stipendiatin aus Indonesien beschäftigte sich im Rahmen ihres zweijährigen Masterstudiengangs Health Care Studies an der Fachhochschule Hamburg intensiv mit dem Gesundheitssystem in Indonesien. Dabei verglich sie das indonesische Gesundheitssystem, das im Jahr 2014 vom indonesischen Staat eingeführt wurde, mit dem deutschen Gesundheitssystem. Sie untersuchte insbesondere was Indonesien bei einer Verbesserung seines noch jungen Systems von Deutschland lernen könnte. Insbesondere die elektronische Verwaltung der staatlich versicherten Patient*innen und die Kostenübernahme für Leistungen niedergelassener Ärtz ${ }^{*}$ innen weisen im indonesischen System ihrer Meinung nach noch erhebliche Mängel auf (Interview mit einer indonesischen Stipendiatin, Skype, 2017). Ein indonesischer Promotionsstudent im Fach Computer Engineering an der TU Dresden arbeitet im Rahmen seiner Doktorarbeit sehr eng mit einer Forscher*innengruppe des Bandung Institute of Technology zusammen. In seiner Arbeit beschäftigt er sich mit der Anwendung von Holz in der indonesischen Architektur. Dabei geht es darum, eine Bauweise von mehrstöckigen Häusern mit Holz zu entwickeln, die beim Auftreten von Erdbeben stabiler sind als herkömmliche Stahl- und Betonhäuser (siehe hierzu noch weiter unten).

\subsection{Tätigkeiten neben dem Studium}

\subsubsection{Berufliche Tätigkeiten}

Einige Stipendiat*innen haben neben dem Studium eine berufliche Tätigkeit ausgeübt. So haben ein paar Alumni vor oder nach dem Stipendium gearbeitet, um sich das Studium zu finanzieren. Auffällig ist dabei, dass dies vor allem bei indonesischen Stipendiat*innen der Fall war und weniger bei georgischen, palästinensischen, ghanaischen und kolumbianischen Stipendiat*innen. Eine indonesische Alumna hatte z. B. während ihres Studiums für eine Consulting-Firma im Baubereich in Karlsruhe gearbeitet. In diesem Nebenjob beriet sie Unternehmen in Bezug auf den Infrastrukturbau in unterschiedlichen Leistungsphasen wie Planung, Durchführung und Dokumentation. Zu den Kund*innen gehörte u. a. die Deutsche Bahn. Obwohl dieser Nebenjob dazu führte, dass sich ihr Studienabschluss um zwei Semester hinauszögerte, war diese Arbeit sehr wichtig für sie und diente als 
Brücke zwischen Studium und Beruf und verhalf ihr zu einem schnellen Berufseinstieg nach dem Studium:

„Der Job war sehr wichtig neben meinem Studium, weil ich dadurch den Job nach dem Studium bekommen habe. Sonst hätte ich den vielleicht nicht bekommen. Da habe ich schon viel über die deutsche Bürokratie und deutsche Gesetze gelernt. Das habe ich später im Beruf auch alles wieder gebraucht" (Interview mit einer indonesischen Alumna in Jakarta, Indonesien, 2017).

Eine andere indonesische Alumna hat ihr viertes Promotionsjahr durch einen Job in einem Callcenter in München finanziert, weil sie das KAAD-Stipendium nur für die ersten drei Jahre der Promotionszeit erhalten hatte. Ein weiterer indonesischer Alumnus arbeite zum Ende seines Diplomstudiums im Jahr 2002 als wissenschaftliche Hilfskraft am Fraunhofer Institut in Berlin. Diese Stelle war auf ein paar Monate befristet.

Vereinzelt haben aber auch Stipendiat*innen aus anderen Ländern einen Nebenjob während des Studiums ausgeübt, als sie noch kein KAAD-Stipendium hatten oder es bereits abgelaufen war und sie noch Möglichkeiten zur Finanzierung des Studiums suchten. Ein ghanaischer Alumnus hatte sich zum Beispiel während des Studiums etwas Geld am Berliner Flughafen als Gepäckträger dazu verdient. Ein anderer ghanaischer Alumnus gründete 2016 neben seinem Studium in Leipzig sogar einen Online-Schuh-Store namens „KOLIKOWEL.com“. In wenigen Ausnahmen haben Alumni während ihres Stipendiums mit einer Sondergenehmigung des KAAD eine Kurzzeittätigkeit neben dem Studium ausgeübt. So hat sich zum Beispiel eine georgische Stipendiatin etwas Geld zu ihrem Stipendium bei McDonalds dazu verdient.

Ein kleiner Teil der Stipendiat*innen hatte zudem ein Praktikum während des Studiums gemacht. So absolvierte ein ghanaischer Stipendiat ein Praktikum bei einer Consultant-Firma in Leipzig, die Unternehmen in Finanz- und Marketingstrategien berät. Eine kolumbianische Alumna machte ein sechsmonatiges Praktikum bei der kolumbianischen Botschaft in Berlin, während ihres Urlaubssemesters an der Pädagogischen Hochschule in Heidelberg.

Als Begründungen für die geringen beruflichen Tätigkeiten neben den Studium führten die Alumni und aktuellen Stipendiat*innen an, dass es zum einen schwer war einen Nebenjob während des Studiums zu finden und zum anderen es im Rahmen des Stipendiums vom KAAD nicht vorgesehen ist, neben dem Studium einer beruflichen Tätigkeit nachzugehen. Zudem wollten sich die meisten der Befragten voll und ganz auf ihr Studium konzentrieren, was eben auch durch die finanzielle Unterstützung des KAAD möglich gemacht wurde. 


\subsubsection{Ehrenamtliche Tätigkeiten}

Die Fokussierung der meisten Stipendiat*innen auf das Studium und wenig Tätigkeiten neben dem Studium spiegelt sich auch bei den eher geringen ehrenamtlichen Tätigkeiten neben dem Studium in Deutschland wider. Weniger als 3\% der befragten Alumni und aktuellen Stipendiat*innen gaben in unserer Onlineumfrage an, sich regelmäßig (monatlich oder wöchentlich) neben ihrem Studium sozial oder politisch in Deutschland engagiert zu haben (nur 10 von 383 befragten Alumni und Stipendiat*innen). Demgegenüber war das kulturelle und religiöse Engagement der Alumni und aktuellen Stipendiat*innen in Deutschland etwas ausgeprägter: Fast ein Drittel der befragten Stipendiat*innen und Alumni war regelmäßig, d.h. wöchentlich oder monatlich, in religiösen Kontexten aktiv (119 von 383 Befragten). Das im Vergleich zum sozialen und politischen relativ hohe religiös-kulturelle Engagement liegt natürlich auch am religiösen Charakter des Stipendienprogramms des KAAD. So ist der KAAD sehr gut mit katholischen Einrichtungen in Deutschland vernetzt. Dazu gehören neben den Ortskirchen in Hochschulstädten vor allem die Katholischen Hochschulgemeinden (KHGs). Die akademischen und kulturellen Angebote der KHG werden auch von KAAD-Stipendiat*innen, die überwiegend katholisch sind, gut angenommen. Im Rahmen unserer Online-Befragung gab fast die Hälfte der Befragten an, regelmäßig in der KHG gewesen zu sein, weitere 40 Prozent der Befragten sagte, zumindest manchmal oder einmal während ihres Studiums in der KHG gewesen zu sein. Nur 11 Prozent gaben an, nie dort gewesen zu sein $(n=390)$. Die Stipendiat*innen gehen in die KHGs entweder zum Mittag- oder Abendessen oder um Freund*innen zu treffen bzw. um an kulturellen Veranstaltungen oder gemeinsamen Messen teilzunehmen oder einfach nur zum Gebet. Ein kolumbianischer Alumnus sagte dazu:

„Ich war bei verschiedenen Veranstaltungen in der KHG. Ich war sehr engagiert. Der Pfarrer dort hat immer internationale Gottesdienste organisiert. Ich war bei Sportaktivitäten dort, Aktionen für Obdachlose, ja, viele Aktivitäten. Das war eine gute Gemeinde" (Interview mit einem kolumbianischen Alumnus in Bogotá, Kolumbien, 2017).

Ein aktueller Stipendiat aus Ghana:

„Ich gehe regelmäßig zum Mittagessen und zu Veranstaltung in der KHG, vor allem internationale Veranstaltungen, auf denen internationale Studenten ihre Länder vorstellen. Ich gehe auch zu den Messen, die von der KHG durchgeführt werden. In München wohne ich in einem katholischen Studentenwohnheim 
mit Priestern und Nonnen, das mir sehr gut gefällt. Ich unterhalte mich gerne mit den Nonnen und Priestern über wichtige gesellschaftliche Fragen, wie zum Beispiel Politik in Deutschland, aber auch in Afrika. Ich habe schon viel durch diese Gespräche gelernt" (Interview mit einem ghanaischen Stipendiaten, Skype, 2017).

Die indonesischen Stipendiat ${ }^{\star}$ innen und Alumni engagieren sich neben der KHG auch sehr stark in der indonesischen Studierendengruppe KMKI („Keluarga Mahasiswa Katolik Indonesia“, „Katholische Indonesische Studentenfamilie“), die bereits vor mehreren Jahrzehnten von katholischen Studierenden aus Indonesien in Aachen gegründet wurde. Eine indonesische Stipendiatin des KAAD, die sichsich als Mitglied bei KMKI engagiert, sagte:

"Ich habe mich bei KMKI engagiert. KMKI ist der größte indonesische Studentenverein in Deutschland. Die sind auch sehr katholisch und machen viele Veranstaltungen. Da wollte ich Mitglied sein und habe auch einige Veranstaltungen in Karlsruhe organisiert. Das hat immer großen Spaß gemacht, auch weil wir alle Indonesier sind, die was erreichen wollen. Zu den christlichen Feiertagen wie Weihnachten waren wir immer zusammen und haben uns über unsere Ideen und Zukunftspläne ausgetauscht. KMKI ist sehr stark unter Naturwissenschaftlern und Technikstudenten. An der Uni Aachen gibt es eine sehr große Gruppe" (Interview mit einer indonesischen Stipendiatin, Skype, 2017).

Die studentischen Aktivitäten von KMKI haben vor allem eine religiöse Ausrichtung wie die Durchführung von heiligen Messen auf Indonesisch, Kreuzwegandachten, Gebets- und Leseabende und gemeinsam organisierte Wallfahrten in Deutschland oder ins europäische Ausland.

Generell zeigte sich eine große Beteiligung an religiösen Veranstaltungen unter den Stipendiat*innen und Alumni. An Gottesdiensten oder anderen religiösen Veranstaltungen in Deutschland (in Kirchen, Moscheen etc.) haben laut unserer Umfrage fast 60 Prozent der Befragten teilgenommen. Rund ein Drittel haben „manchmal“ teilgenommen, etwas über zwei Prozent einmal und nur knapp fünf Prozent überhaupt nicht ( $\mathrm{n}=390)$.

Insgesamt muss man aber sagen, dass die Stipendiat ${ }^{*}$ innen und Alumni darüber hinaus nur wenig am allgemeinen sozialen Leben in Deutschland teilhaben. Vielmehr bleiben sie in ihrer Freizeit untereinander, also zumeist zusammen mit anderen Stipendiat*innen des KAAD oder Studierenden aus denselben Herkunftsländern. Ein ghanaischer KAAD-Stipendiat organisierte zum Beispiel Business-Seminare für 
andere KAAD-Stipendiat ${ }^{*}$ innen, in denen sich die Stipendiat ${ }^{*}$ innen über Job- oder Businessideen in Deutschland und in den Herkunftsländern austauschen konnten. Dafür reiste er zwei Mal zurück nach Ghana, um die Seminare in Kumasi vorzubereiten und mit Kolleg*innen durchzuführen. Zudem merkten die interviewten Alumni häufig an, dass es schwierig ist, sich in Deutschland gesellschaftlich zu engagieren. Ein befragter ghanaischer Alumnus sagte dazu:

„Wo sollte ich das machen. Die deutschen Vereine waren für mich ziemlich geschlossen. Außerdem wird in den deutschen Organisationen nur Deutsch gesprochen. Für einen Ausländer ist es sehr schwierig da reinzukommen" (Interview mit einem ghanaischen Alumnus in Deutschland, Skype, 2018).

Eine andere Stipendiatin aus Ghana meinte:

„Für ein gesellschaftliches oder religiöses Engagement habe ich leider keine Zeit. Das Studium erfordert alles von mir" (Interview mit einer ghanaischen Stipendiatin, Skype, 2017).

\subsection{Herausforderungen in Studium und Alltag}

Es ist nicht überraschend, dass die Alumni einige Herausforderungen gerade zu Beginn ihrer Studien- und Forschungszeit in Deutschland zu bewältigen haben. Dazu gehören vor allem das Erlernen einer neuen Sprache, der Umgang mit einer neuen Kultur und das Einleben in eine Hochschullandschaft, die sich zumeist erheblich von den Hochschulen unterscheidet, die die Studierenden aus den Herkunftsländern kennen. All diese verschiedenen Herausforderungen, die im Alltags- und Universitätsleben gleichzeitig auf die Studierenden zukommen, müssen zur gleichen Zeit bewältigt werden. Eine Aussage eines indonesischen Alumnus bringt dies auf den Punkt:

„Ich lebte wie in zwei Welten. Ich war in Gedanken noch in Indonesien und bei meinem Alltagsleben in Jakarta. Gleichzeitig musste ich aber alles managen in Deutschland, Studium, Wohnung, Freizeit und Behördengänge. Das war sehr schwierig am Anfang das alles unter einen Hut zu bekommen"(Interview mit einem indonesischen Alumnus in Jakarta, Indonesien, 2017). 
Zudem wurden die Alumni mit Herausforderungen konfrontiert, die sie vorher im Herkunftsland nicht vorhergesehen haben bzw. erwartet haben. Ein zentrales Problem für die Alumni, über das sie sich vorher nicht bewusst waren, war die ,deutsche Mentalität' und die kulturellen Gepflogenheiten in Deutschland, die sehr fremd für sie waren und sehr „kühl“ auf sie wirkten. Folgende Zitate von verschiedenen Alumni mach dies deutlich:

„Die deutsche Mentalität, eigentlich die gesamte Kultur, war ein Problem. Ich konnte nicht verstehen, warum die Deutschen so kühl und distanziert waren, nicht nur zu Fremden, sondern auch untereinander. Man hat nur wenig Spaß miteinander gehabt. Das habe ich mich schon sehr alleine manchmal gefühlt" (Interview mit einer ghanaischen Alumna in Accra, Ghana, 2018).

"Ich hatte große Probleme mich mit den Deutschen zu verständigen. Sie erschienen mir immer sehr distanziert und wenig interessiert. Auch mir war die deutsche Sprache und Kultur sehr befremdlich am Anfang, weil einfach alle Regeln in der Kommunikation und im Umgang miteinander anders waren als in Ghana. Es wurde weniger gesprochen und miteinander gegessen und gefeiert. Es lag aber auch mit an mir, denn ich war am Anfang auch sehr schüchtern und konnte nur schwer auf die Leute zugehen. Ich hatte am Anfang eigentlich nur Zeit mit den anderen Stipendiaten aus Afrika verbracht. Das hat mir auch geholfen über die schwierige Anfangszeit hinwegzukommen. Das ging allen von uns am Anfang so. Das hat sich erst im Laufe des Studiums geändert, als ich mich mehr geöffnet habe und auch die deutschen Kommilitonen, die ähnliche Interessen im Studium wie ich hatten, auf mich zugekommen sind." (Interview mit einem ghanaischen Alumnus in Kumasi, Ghana, 2018).

"Die gegenseitige Distanz der Deutschen war für mich das größte Problem“ (Interview mit einer kolumbianischen Alumna in Bogotá, Kolumbien, 2017).

"It was difficult to learn German, of course. But it was also difficult to make friends with Germans. It can take really long, and sometimes you even don't get in touch with them. Germans are very closed, and I had only friends, who were also KAAD scholars. Later I got also some German friends, but it took over one year. I guess that is a special German mentality, much different than our mentality in Indonesia" (Interview mit einer indonesischen Alumna in Jakarta, Indonesien, 2017). 
Die distanzierte Kommunikationsweise der Deutschen machte sich auch im wissenschaftlichen Bereich für die Alumni bemerkbar. Ein ghanaischer Stipendiat äußerte sich dazu:

„Es war sehr schwer am Anfang, sich auf das akademische Arbeiten in Deutschland einzustellen. Gerade als Doktorand arbeitet man hier sehr alleine. In Ghana arbeitet man häufiger im Team und ist im ständigen Austausch mit den anderen Doktoranden und dem Betreuer. Daran musste ich mich erst einmal gewöhnen, dass das hier so anonym ist" (Interview mit einem ghanaischen Stipendiaten, Skype, 2017).

Die Alumni bedauerten, dass es sehr schwierig ist und sehr lange dauern kann, bis man eine Freundschaft mit Deutschen aufgebaut hat:

„Es ist sehr schwer Freundschaften mit Deutschen aufzubauen, oder Netzwerke aufzubauen. Das dauert sehr lange in Deutschland, weil die Deutschen sehr distanziert sind. In Kolumbien ist alles mehr auf emotionaler Ebene. Dort muss man auch über private Dinge sprechen, um eine Beziehung aufzubauen, auch an der Arbeit. Und man muss Dinge zusammen unternehmen, damit man sich besser kennenlernt und miteinander arbeiten kann. Es ist überhaupt nicht schlimm über private Dinge an der Arbeit zu sprechen. [...] Ich würde sagen, ich habe keine deutschen Freunde, sondern nur Bekannte. In Heidelberg mache ich mehr Sachen mit meinen polnischen Freundinnen zusammen, wie Volleyball spielen, oder ich treffe mich mit einer älteren kolumbianischen Freundin zu Kaffee trinken in der Heidelberger Altstadt. [...] Ich habe öfter Heimweh nach Kolumbien. Ich vermisse vor allem meine Freunde und meine Familie in Medellin" (Interview mit einer kolumbianischen Alumna in Deutschland, Heidelberg, 2018).

Eine andere kolumbianische Alumna hat es ähnlich drastisch formuliert:

„Ich glaube, wir hätten keine sehr guten Jobaussichten gehabt. Außerdem waren die alltäglichen Herausforderungen gerade für mich zu groß. Ich hatte große Probleme mich an das Klima anzupassen, die Leute waren zu schwer, sehr distanziert. Ich habe das irgendwie akzeptiert, so nach drei Jahren. Ich hatte auch nur eine richtige deutsche Freundin in über fünf Jahren, sonst waren meine Bekanntschaften alle eher aus Lateinamerika. Wir sind in der Mentalität einfach zu verschieden. Mein Mann konnte damit besser umgehen. Der hatte auch einige deutsche Freunde. Aber vielleicht sind Männer da anders. 
[...] Ich konnte keine guten Gastgeber in Deutschland finden. Ich hatte wenig Kontakt zu Deutschen, aber das liegt auch ein bisschen an mir, weil ich sehr schüchtern bin"(Interview mit einer kolumbianischen Alumna in Medellin, Kolumbien, 2017).

Ein ghanaischer Stipendiat bemängelte vor allem die fehlende Unterstützung durch seine deutschen Kolleg*innen und fühlte sich häufig alleine gelassen:

„Die Anonymität in der Wissenschaft: Man ist doch sehr auf sich allein gestellt. Das ist im Alltag auch so. Es gibt viele Individualisten in Deutschland, aber wenig Team-Player. Ich glaube, ich wäre noch besser und noch schneller in meiner Doktorarbeit, wenn ich mehr Unterstützung bekommen würde. Manchmal würde es schon reichen, wenn ich mich mit einem Kollegen über meine Arbeit austauschen könnte, oder einfach mal über Gott und die Welt zu plaudern" (Interview mit einem ghanaischen Stipendiaten, Skype, 2017).

Andere formulierten es ähnlich:

„Vor allem die Einsamkeit war ein großes Problem in Deutschland für mich. Es war schwer deutsche Freunde zu bekommen [...] In Deutschland habe ich mich unwohler gefühlt als in den Niederlanden. Man kann das an einer Situation verdeutlichen, die mir so in den Niederlanden vorher nicht passiert ist: Als ich 2008 in Deutschland ankam, habe ich einen älteren Mann auf der Straße nach dem Weg zu einem Internetcafé gefragt, aber er hat nicht geantwortet, sondern ist weggelaufen. Dann kam er wieder zurück und mir einen Euro gegeben. Den habe ich nicht angenommen, und habe mich sehr unwohl in dieser Situation gefühlt"(Interview mit einer ghanaischen Alumna in Accra, Ghana, 2018).

Dabei wird häufig auch die mangelnde Spontanität unter den Deutschen kritisiert:

„In Deutschland kannst du nicht einfach einen Freund oder Bekannten spontan besuchen. Nein. Sondern du musst dich erst vorher anmelden, du nicht einfach zu ihnen ins Haus gehen, sie müssen sich erst auf deinen Besuch vorbereiten. Das ist bei uns in Ghana ganz anders, da kannst Du immer einfach und spontan vorbeikommen. Man sieht dann einfach, was man zusammen macht"(Interview mit einem ghanaischen Alumnus in Accra, Ghana, 2018). 
Viele internationale Studierende - egal ob sie aus Georgien, Ghana, Palästinma, Indonesien oder Kolumbien kommen - nehmen zudem das berufliche und alltägliche Miteinander in Deutschland als „zu konkurrenzorientiert“ wahr. Eine kolumbianische Alumna hat das in den Seminaren an der Universität als ein einschneidendes Erlebnis wahrgenommen und formulierte es drastisch:

„Die Deutschen sind sehr wettbewerbs- und konkurrenzorientiert. Ich war schockiert, dass sogar meine Kommilitonen in den Seminaren an der Hochschule sehr auf sich bezogen sind. Das Klima war sehr rau und kalt, und jeder wollte der Beste sein. Das hat mir gar nicht gefallen. Auch weil das noch sehr junge Leute erst zwischen 20 und 25 Jahre alt waren, viel Jünger als ich. Die haben sich voneinander abgegrenzt, Gruppen gebildet und andere ausgeschlossen. Sie wollten dadurch besser werden und bessere Noten bekommen. Man hat sich nicht untereinander geholfen. Da waren die Deutschen in einer Gruppe unter sich, und auch die türkischen Studenten in einer Gruppe unter sich. Das habe ich nicht verstanden, und kannte ich auch nicht. Auch ich wurde öfter ausgegrenzt. Auch weil ich eine Latina bin. Da habe ich mich manchmal alleine gefühlt, auch diskriminiert. Solchen Wettbewerb mag ich nicht, wir sollten mehr zusammen lernen und uns gegenseitig helfen. Den Deutschen fehlt auch manchmal Empathie, vor allem gegenüber Leuten aus anderen Kulturen" (Interview mit einer kolumbianischen Alumna in Deutschland, Heidelberg, 2018).

Auch die anfänglichen Behördengänge, u. a. zur Registrierung beim Einwohnermeldeamt oder zur Einschreibung bei der Universitätsverwaltung, bereiteten den Alumni einige Schwierigkeiten, weil sie viele Dokumente, die auf Deutsch verfasst waren, nicht verstanden haben. Auch eine geeignete Wohnung bzw. ein geeignetes Zimmer zu finden, stellte sich manchmal ebenfalls als problematisch für die Alumni dar. Eine kolumbianische Alumna dazu:

"It was very difficult to find an apartment in Duisburg. The first month I was in a small apartment alone, but I didn't like it, it was quite expensive, and I felt lonely. Then I could move into an apartment that I shared with other students from other countries in a Studentenwohnheim. I liked that much more, because I was not so alone, and we did a lot of things together, like cooking, running, sports, and watching movies" (Interview mit einer kolumbianischen Alumna in Bogotá, Kolumbien, 2017). 
Stipendiat ${ }^{\star}$ innen, die eigene Kinder und eine Ehefrau oder einen Ehemann in ihrem Heimatland zurücklassen mussten und alleine nach Deutschland zum Studieren gekommen sind, hatten noch größere Probleme, weil sie natürlich ihre Familie vermissten und sich so gut es eben ging aus der Distanz um sie kümmern mussten. Ein Alumnus aus Ghana berichtete, dass es ihm am Anfang extrem schwerfiel, ohne seine Ehefrau und zwei kleinen Töchter in Deutschland zu leben. Dass er seine Familie nicht mit nach Deutschland bringen durfte, war eine Voraussetzung, um das KAAD-Stipendium zu bekommen. Das wurde damit begründet, dass ein Stipendium nicht die Lebenshaltungskosten für eine vierköpfige Familie abdecken würde und es die Rückkehrbereitschaft des Stipendiaten nach der Promotion wahrscheinlich verringern würde. Der Alumnus sagte dazu:

„Die Kälte und die Dunkelheit, das hat mich fast depressiv gemacht, und dann hat mir natürlich meine Familie noch mehr gefehlt. Mit dem Frühling und im Sommer wurde es besser" (Interview mit einem ghanaischen Alumnus in Deutschland, Skype, 2017).

Ähnlich ging es auch einem anderen ghanaischen Stipendiaten, der sogar drei Kinder und seine Ehefrau in Ghana zurücklies, die daraufhin zu seinen Eltern und seinem Bruder in die Stadt Wa im Norden Ghanas zogen (Interview mit einem ghanaischen Stipendiaten, Skype, 2017).

Ein anderer Alumnus aus Kolumbien durfte seine Ehefrau nach Deutschland mitbringen und hatte dafür auch zusätzliche finanzielle Unterstützung vom KAAD bekommen. Dennoch war es schwierig mit dem Geld über die Runden zu kommen:

"Ich bin mit meiner Frau nach Deutschland gekommen. Das hat mir auch beim Übergang am Anfang geholfen. Der KAAD hat uns deswegen auch 500 Euro extra gezahlt, also insgesamt haben wir 1.500 Euro vom KAAD bekommen, und die Krankenversicherung wurde aber nur zu 60 Prozent vom KAAD zusätzlich gezahlt. Trotzdem war das Geld noch nicht genug und wir konnten uns auch keine Wohnung wie in Bogotá leisten, sondern nur eine sehr kleine Einzimmerwohnung etwas außerhalb von Frankfurt. Alleine für die Miete haben wir 550 Euro bezahlt. Bis zur Uni habe ich fast eine Stunde gebraucht. Das war schon schwierig, auch beim Essen mussten wir immer rechnen. Als dann später unsere kleine Tochter noch dazu kam, wurde es noch schwieriger uns ausreichend zu finanzieren" (Interview mit einem kolumbianischen Alumnus in Bogotá, Kolumbien, 2017). 
Demgegenüber gab es aber auch einige Stipendiat*innen, die keine großen Anpassungsprobleme zu Beginn ihrer Zeit in Deutschland hatten. Eine Stipendiatin aus Ghana sagte, dass ihr die Eingewöhnungsphase in Deutschland sogar sehr leichtfiel, weil ihr Ehemann bereits in Deutschland war und er sie dabei gut unterstützt hat. Sie konnten von Beginn an zusammen in einer Mietwohnung in Bayreuth, wo beide studiert haben, wohnen. Ähnlich ging es auch einer kolumbianischen Alumna:

„Im kam im März 2007 nach Deutschland. Es hat geholfen, dass mein Mann schon ein halbes Jahr an der Uni Gießen promoviert hat, weil es dann auch mir leichter fiel, mich dort einzuleben. Ich hatte ja überhaupt keine Ahnung von Deutschland. Das war alles sehr neu für mich, das Wetter, das Essen, die Mentalität, alles. [...] Das erste Jahr mit dem Intensivsprachkurs war schon hart, die Sprache ist extrem schwer und ich musste Tag und Nacht lernen. Das war eine neue Welt für mich" (Interview mit einer kolumbianischen Alumna in Medellin, Kolumbien, 2017).

Zudem half einigen Alumni auch die besondere Situation und die Freude und Dankbarkeit in Deutschland zu sein über die anfänglichen Herausforderungen und Probleme hinweg zu kommen. So erinnert sich zum Beispiel ein Alumnus an die aufregende Zeit in Berlin unmittelbar nach dem Fall der Berliner Mauer, während er sein Studium in Berlin anfing. Dieses weltgeschichtliche Ereignis hautnah mitzuerleben, half ihm bei der Bewältigung seines anfänglichen Heimwehs und seiner Probleme mit der „kalten Mentalität“ der Deutschen (Interview mit einem ghanaischen Alumnus in Accra, Ghana, 2018). Letztendlich konnten die meisten Alumni die anfänglichen Schwierigkeiten jedoch überstehen und ihr Studium oder ihren Forschungsaufenthalt in Deutschland erfolgreich durchführen. Eine Alumna aus Kolumbien brachte diese Erkenntnis, die auch einen wichtigen Teil der Erfahrungen in Deutschland ausmacht, folgendermaßen auf den Punkt:

„Ja, es gab natürlich auch viele Schwierigkeiten, wie die Ernährung, das Klima, die Kultur. Wenn mir jemand sagt, dass er im Ausland studieren möchte, dann sage ich immer, ja, die erste Zeit ist die schlimmste, aber dann gibt es keine Probleme mehr. Wenn man erst einmal die kulturellen Codes verstanden hat, dann geht es. Am Anfang ist das erst einmal sehr kompliziert. Es gibt kein Handbuch für die deutsche Kultur von A bis Z. Das ist mit anderen Kulturen genauso, bevor es gut läuft" (Interview mit einer kolumbianischen Alumna in Bogotá, Kolumbien, 2017). 
Eine weitere große Herausforderung stellte, wie oben angedeutet, die deutsche Sprache dar, die von vielen Stipendiat ${ }^{\star}$ innen und Alumni nur sehr schwer erlernt werden konnte oder während des gesamten Studiums oder der Promotion überhaupt nicht gelernt wurde. Ein aktueller Stipendiat monierte:

"Die deutsche Sprache macht mir immer noch große Probleme. Obwohl ich einen Sprachkurs in Bonn besucht habe und auch in meiner Freizeit viel deutsch sprechen muss, kann ich immer noch nicht so richtig sprechen. Meine Doktorarbeit schreibe ich auf Englisch, das ist ein Glück" (Interview mit einem ghanaischen Stipendiaten, Bonn, 2017).

Teilweise versuchen Stipendiat ${ }^{\star}$ innen das Erlernen der deutschen Sprache auch zu umgehen, indem sie englischsprachige Studiengänge oder Seminare wählen und ihre Master- und Doktorarbeiten auf Englisch verfassen. Wenn sie sich dann doch einmal auf Deutsch im Rahmen ihrer Master- oder Doktorarbeit verständigen müssen, kommt es oftmals zu Schwierigkeiten, wie das folgende Beispiel eines kolumbianischen Alumnus illustriert:

„Die Doktorandenkolloquien waren auf Englisch, das war immer sehr gut und hilfreich. Da habe ich immer ein sehr gutes Feedback bekommen. Die Verteidigung der Doktorarbeit war aber auf Deutsch, das war nicht so gut, und da hatte ich auch große Probleme, die Fragen gut und richtig zu beantworten" (Interview mit einem kolumbianischen Alumnus in Bogotá, Kolumbien, 2017).

Ein anderer Kolumbianer brachte es abschließend schlicht und einfach auf den Punkt:

"Deutsch ist zu schwierig“ (Interview mit einem kolumbianischen Stipendiaten, Skype, 2017).

Viele Alumni schrieben ihre Master-, Diplom- oder Doktorarbeit daher auf Englisch. Das hatte häufig ganz pragmatische Gründe: Zum einen fiel es vielen Alumni leichter auf Englisch zu schreiben als auf Deutsch. Zum anderen wählten sie Themen für ihre Abschlussarbeiten, die sich auch auf das Herkunftsland (oder sogar ausschließlich auf das Herkunftsland) bezogen und somit auch für Wissenschaftler ${ }^{\star}$ innen in den Herkunftsländern von Interesse waren. Oder sie wählten Themen, die zwar nichts mit dem Herkunftsland zu tun hatten, aber generell für eine internationale Leser*innenschaft interessant sein könnten. Dafür eignet sich die englische besser als die deutsche Sprache. 
Auch mit finanziellen Problemen hatten ein paar Alumni im Rahmen ihres KAAD-Stipendiums zu kämpfen. Insbesondere wenn Stipendiat ${ }^{*}$ innen mit ihrer Familie in Deutschland leben, für die zusammen ein Stipendium trotz familienbezogener Zusatzzahlungen nicht ausreicht:

"Ich bin mit meiner Frau nach Deutschland gekommen. Das hat mir auch beim Übergang am Anfang geholfen. Der KAAD hat uns deswegen auch 500 Euro extra gezahlt, also insgesamt haben wir 1.500 Euro vom KAAD bekommen, und die Krankenversicherung wurde aber nur zu 60 Prozent vom KAAD zusätzlich gezahlt. Trotzdem war das Geld noch nicht genug und wir konnten uns auch keine Wohnung wie in Bogotá leisten, sondern nur eine sehr kleine Einzimmerwohnung etwas außerhalb von Frankfurt. Alleine für die Miete haben wir 550 Euro bezahlt. Bis zur Uni habe ich fast eine Stunde gebraucht. Das war schon schwierig, auch beim Essen mussten wir immer rechnen. Als dann später unsere kleine Tochter noch dazu kam, wurde es noch schwieriger uns ausreichend zu finanzieren. [...] Weil wir so wenig Geld hatten, sind wir auch nur wenig in Deutschland gereist. Dafür konnte ich mich auf meine Doktorarbeit konzentrieren. Trotzdem war es ein großer Stress, dass ich nicht genug Geld hatte. Wir sind nie ins Restaurant gegangen, haben nur im Aldi eingekauft und zu Hause gekocht" (Interview mit einem kolumbianischen Alumnus in Bogotá, Kolumbien, 2017).

Die Stipendiat ${ }^{\star}$ innen und Alumni berichteten auch von Diskriminierungserfahrungen. Diese machten sie weniger an der Universität oder im Arbeitsleben als vielmehr in alltäglichen Situationen auf der Straße, im Bus oder im Supermarkt. Ein kolumbianischer Alumnus berichtete:

„Diskriminierung ist eine Einstellung von vielen Leuten, die entscheiden wer wichtig ist und wer nicht. Für mich wäre es ganz schlimm gewesen, wenn meine Kollegen in der Kanzlei oder wenn meine Kommilitonen an der Universität mich als Lateinamerikaner diskriminiert hätten. Und diese Erfahrung habe ich nie gehabt. Diskriminierung habe ich mehr im Bus gefühlt, oder im Supermarkt. [...] Sie wollten nicht mit mir sprechen. Punkt. Und ich bin manchmal darauf angewiesen von den Leuten angesprochen zu werden, oder dass die Leute mir eine Antwort geben. Zum Beispiel war ich in einem Supermarkt alleine. Wenn ich einen Joghurt kaufe, frage ich eine Person in meiner Nähe was ist das für ein Joghurt? Ich merkte, dass es viele Leute gab, die mit mir nicht sprechen wollten. Aber nicht etwas sehr Schlimmes. Aber insgesamt als Südamerikaner ist gut, die Deutschen mögen die Südamerikaner. Insgesamt 
ist alles gut" (Interview mit einem kolumbianischen Alumnus in Bogotá, Kolumbien, 2017).

Ein Alumnus aus Ghana:

„Vor allem im Bus konnte ich spüren, dass sich Menschen eher weg von mir setzen, oder sie haben es sogar bevorzugt zu stehen, obwohl neben mir Plätze frei sind. Das hat mich schon öfter verletzt. Das ist sogar häufig passiert" (Interview mit einem ghanaischen Alumnus in Accra, Ghana, 2018).

Ein anderer ghanaischer Alumnus machte ähnliche Erfahrungen:

"Ich habe auch manchmal Rassismus in Deutschland gespürt. Am Anfang während des Deutschkurses in Deutschland nicht so sehr, aber später in Bremen ist mir das aufgefallen, vielleicht weil die Stadt weniger international ist. [...] Mir fällt keine besonders dramatische Situation ein, in der ich als schwarzer Mann direkt beschimpft oder attackiert wurde, aber in vielen kleinen Situationen des Alltags konnte ich es merken. An der Supermarktkasse oder bei Behördengängen wurde mein Geld mehrmals nachgezählt, um zu sehen, ob ich den richtigen Betrag zahle, oder meine Dokumente wurden häufiger auf Richtigkeit geprüft als bei weißen Leuten. Also ein gewisses Misstrauen mir gegenüber konnte ich häufig spüren" (Interview mit einem ghanaischen Alumnus in Kumasi, Ghana, 2018).

Ein anderer Rassismusfall eines ghanaischen Alumnus war sogar lebensbedrohlich:

„Als ich nach einer Grillparty der Ghana Association in Berlin auf dem Heimweg war, haben mich mehrere Skinheads verfolgt. In der U-Bahn haben sie mich angeschrien und bedroht. Einer hat mir dann eine Bierflasche an den Kopf geworfen und ich blutete. Dieses Erlebnis hat mir so viel Angst eingejagt, dass ich sofort danach meine Sachen gepackt habe und nach Hause fliegen wollte. Meine WG-Mitbewohner haben mich aber überredet in Deutschland zu bleiben. Sie haben gesagt, dass es das nicht wert sei und ich nicht meinen Master aufs Spiel setzen solle. Ich bin letztendlich geblieben und habe meinen Abschluss gemacht, aber ich war wirklich kurz davor, alles hinzuschmeißen. Das war in den 90er Jahren in Berlin" (Interview mit einem ghanaischen Alumnus in Accra, Ghana, 2018). 
Er machte auch weitere alltägliche Erfahrungen von Rassismus:

„Auch während meiner Arbeit am Flughafen Tegel wurde ich viel beschimpft, meistens als Neger. Auch Kinder haben mich als Neger beschimpft. Das sind Kinder und ich mache sie nicht dafür verantwortlich, sie haben das von den Erwachsenen gehört. Aber es hat mich trotzdem traurig gemacht" (Interview mit einem ghanaischen Alumnus in Accra, Ghana, 2018).

Interessanterweise haben die Stipendiat*innen und Alumni kaum Diskriminierung in Deutschland erfahren, die auf ihre Religion abzielte. In der Umfrage gaben rund 85 Prozent an, dass sie sich nie wegen ihres religiösen Glaubens in Deutschland diskriminiert gefühlt haben. Weitere elf Prozent haben zumindest selten diese negative Erfahrung gemacht. Knapp drei Prozent der Befragten fühlten sich häufig oder sehr häufig diskriminiert $(\mathrm{n}=390)$.

Zudem hatten Stipendiat*innen und Alumni auch mit privaten und familiären Problemen zu kämpfen. Eine kolumbianische Alumna konnte ihre Doktorarbeit lange Zeit nicht abschließen, weil sie mit mehreren privaten Schicksalsschlägen fertig werden musste:

„Ich hatte so viele Probleme. Ich musste viel nebenher arbeiten und ich hatte einen Sohn hier in Kolumbien, und gleichzeitig meine Dissertation. Zudem wurde ich krank und ich musste operiert werden. Und mein Vater ist auch noch in der Zeit gestorben. Also es gab so viele Probleme. Und das war auch ein Grund zurückzukommen. Ich fühlte mich sehr alleine in Deutschland. Und es gab auch familiäre Probleme und ich hatte das Bedürfnis noch einmal mit der Familie zusammen zu sein. In dieser Zeit war ich an der HumboldtUniversität in Berlin. Das war ein internationales Studienprogramm in einer Graduate School"(Interview mit einem kolumbianischen Alumnus in Accra, Ghana, 2018).

Eine aktuelle Stipendiatin muss neben dem Studium eine kleine Tochter großziehen:

„Das Studium und sich nebenbei um meine kleine Tochter zu kümmern ist sehr schwierig. Manchmal kann ich mich nicht auf meine Hausaufgaben zu Hause konzentrieren, weil meine kleine Tochter spielen möchte" (Interview mit einer ghanaischen Stipendiatin, Skype, 2017). 


\subsection{Erwerb von Fähigkeiten in Deutschland}

Die befragten Stipendiat*innen und Alumni haben nach eigenen Aussagen viele Fähigkeiten (Skills) in Deutschland erworben, die sie selbst auch als eine wichtige Ressource für ihren weiteren Karriereweg einschätzen. $\mathrm{Zu}$ den meistgenannten Fähigkeiten gehören neben den Sprachkenntnissen ${ }^{11}$, berufliches Fachwissen, interkulturelle Kompetenzen, neue Arbeitstechniken und das Kennenlernen einer neuen Arbeitsethik. In der Online-Umfrage antworteten über 80 Prozent der Befragten, dass sie „sehr viele“ oder „viele“ berufliche Fähigkeiten während des Studiums in Deutschland erwarben $(\mathrm{N}=378)$.

Auch in den Interviews mit Stipendiat*innen und Alumni aus den fünf Fallstudienländern wurde der Erwerb von beruflichen Fachkenntnissen als wichtigster Bildungserwerb während des Studiums in Deutschland angesehen. Eine ghanaische Alumna, die während ihres Studiums die neuesten Technologien für die Vermessung von Landflächen kennengelernt hat, betonte:

„Solche Ausbildungsmöglichkeiten hat man in Ghana nicht, obwohl sie dringend benötigt werden und eigentlich auch die Unternehmen danach fragen und die Regierung Aufträge dafür vergeben will“ (Interview mit einer ghanaischen Alumna in Accra, Ghana, 2018).

Und fügte an: „Ich wurde eine gebildete Frau in Deutschland.“ Eine aktuelle Stipendiatin aus Ghana brachte es folgendermaßen auf den Punkt:

„Das Studium in Deutschland ist sehr wichtig. Ich habe mich hier doppelt so schnell entwickelt wie in Ghana. Das lag vor allem daran, dass ich hier einen schnellen Zugang zu Wissen habe, vor allem Bücher und alle Fachpublikation kostenlos online. Das wird an vielen Unis in Ghana nicht angeboten" (Interview mit einer ghanaischen Stipendiatin, Skype, 2017).

11 Knapp drei Viertel der befragten Stipendiat*innen und Alumni antworteten, dass sie „sehr intensiv“ oder „intensiv“ die deutsche Sprache erworben hätten. Demgegenüber antworteten fast 20 Prozent der Befragten „etwas“ und rund sieben Prozent „wenig“. Nur ein Prozent antwortete, dass es überhaupt keine deutsche Sprache erlernt hätte $(\mathrm{N}=378)$. Zu den Alumni, die nur etwas oder kein Deutsch gelernt haben, gehören auch diejenigen, die in einem englischsprachigen Studiengang studiert oder ihre Doktorarbeit auf Englisch (ferner auch auf Spanisch) verfasst haben. 
Für einen kolumbianischen Alumnus waren die Schreibfertigkeiten, die er sich während der Promotion in Deutschland aneignete, die brauchbarsten Fähigkeiten für seinen späteren akademischen Weg:

„Deutschland hat mein Leben komplett verändert. Alleine in Deutschland zu leben und dort die Doktorarbeit zu schreiben, kann ein großer Vorteil für die akademische Karriere in Kolumbien sein. Durch das Schreiben meiner Doktorarbeit habe ich gelernt analytisch zu denken und mich über einen längeren Zeitraum auf eine Sache intensiv zu konzentrieren. Außerdem habe ich gelernt, interkulturell zu kommunizieren. Das sind wichtige Skills, um ein internationales Netzwerk aufzubauen und um Fundraising machen zu können" (Interview mit einem kolumbianischen Alumnus in Bogotá, Kolumbien, 2017).

Eine kolumbianische Psychologin lernte in ihrem Studium neue Zweige in ihrem Fach kennen:

„Zum Beispiel war es für meinen späteren Beruf sehr wichtig, zu sehen, dass man sich in der Psychologie auf die Betreuung von Kindern spezialisieren kann, also dass es spezielle psychologische Behandlungsmethoden von psychisch eingeschränkten Kindern oder Kindern mit Aufmerksamkeitsdefiziten gibt, und dass es dafür auch spezielle Behandlungsmethoden gibt. In Kolumbien gibt es so was nicht, da gehen die Eltern, wenn überhaupt, mit ihren Kindern zu allgemeinen Psychologen, die keine Ausbildung für die Behandlung von Kindern haben und eigentlich auch überhaupt nicht mit Kindern umgehen können" (Interview mit einer kolumbianischen Alumna in Medellin, Kolumbien, 2017).

Ähnlich positive Erfahrungen machte auch ein kolumbianischer Jurist:

„Was ich in Deutschland noch gelernt habe, war, wie konnte ich meinem Land mit meiner Arbeit helfen. Ich glaube, das war sehr wichtig. Außerdem Disziplin, methodisches Denken und Arbeiten, juristische Sachen. Und ich habe den Bundesgerichtshof in Karlsruhe besucht, und ich habe mit vielen Richtern und wissenschaftlichen Mitarbeitern gesprochen. Das war eine sehr interessante Erfahrung, um viele Beispiele kennenzulernen, und um meine Arbeit hier in Kolumbien zu verbessern" (Interview mit einem kolumbianischen Alumnus in Bogotá, Kolumbien, 2017).

Als zweitwichtigste Fähigkeit, die man in Deutschland erlangte - nach den akademischen und beruflichen Fähigkeiten - wurden interkulturelle Kompetenzen von 
den befragten Stipendiat ${ }^{\star}$ innen und Alumni genannt. In unserer Onlinebefragung antworteten ebenfalls über 80 Prozent, dass sie „sehr viel“ oder „viel“ an interkultureller Kompetenz in Deutschland erworben hatten. Weitere 15 Prozent sagten, „etwas" und nur etwas über drei Prozent entweder „wenig“ oder „gar nicht“ ( $(n=378)$.

Viele Alumni sehen Deutschland demnach als ein kulturell vielfältiges Land, in dem sie mit Menschen aus vielen Ländern in Kontakt kamen und dabei verschiedene Kulturen kennenlernten. Diese Möglichkeit haben sie in ihren Herkunftsländern meistens nicht gehabt. Eine Kolumbianerin sagte dazu:

„Sehr intensiv. Ich kann mein Leben in zwei Teile teilen: Mein Leben vor Deutschland und mein Leben nach Deutschland. Ich [bekam] sehr viel Hilfe, von meiner Uni, vom KAAD, ich habe wunderbare Leute kennengelernt. Aber es war eine ganz andere Kultur und ich konnte meine Augen groß öfnen. Deutschland ist ein Land mit Geschichte von Migration, natürlich nicht wie in den USA, aber ich konnte Leute von der ganzen Welt in Deutschland kennenlernen. Diese Möglichkeit hat man hier nicht. Kolumbien ist ein Land mit wenig Immigration aus anderen Ländern außerhalb Lateinamerikas. Und in den 1930er Jahre wurde Einwanderung in Kolumbien verboten. Deshalb haben wir wenig Erfahrung mit anderen Kulturen. Nur Immigration aus unseren Nachbarländern, wie zurzeit aus Venezuela. Wir sind nicht wie Brasilien oder Argentinien. Deswegen war Deutschland für mich die beste Möglichkeit die Welt durch die Leute in Deutschland kennenzulernen. [...] Und ich hatte das Glück mit meinem Professor und Leuten aus anderen Ländern, Ukraine, Taiwan, Mexiko, Albanien, Türkei. Also die Möglichkeit mit Menschen aus der ganzen Welt zu forschen, da habe ich natürlich viel gelernt" (Interview mit einer kolumbianischen Alumna in Bogotá, Kolumbien, 2017).

Ein ghanaischer Alumnus wurde durch den Kontakt und Austausch mit Menschen aus anderen Ländern für andere Kulturen sensibilisiert:

„Eine wichtige Lehre für mich war auch, jede Kultur erst einmal zu akzeptieren und versuchen sie zu verstehen und nicht zuerst kritisieren. Jede Kultur ist anders, und zuerst muss man erst einmal Informationen über die neue Kultur sammeln. Zu Beginn hat man zu wenig Informationen, erst wenn man genügend Informationen über die andere Kultur hat, kann man sich eine Meinung bilden"(Interview mit einem ghanaischen Alumnus in Accra, Ghana, 2018).

Darüber hinaus lernten die Stipendiat ${ }^{\star}$ innen und Alumni auch eine neue Arbeitsweise in Deutschland kennen. Rund ein Drittel der Befragten in unserer Online-Be- 
fragung gab an, „sehr viel“ oder „viel“ über eine neue Arbeitsweise gelernt zu haben. Weitere 19 Prozent haben „etwas“ über die Arbeitsweisen in Deutschland gelernt. Ein kolumbianischer Alumnus erklärte diese neue Arbeitsweise folgendermaßen:

„In Kolumbien sind wir immer sehr ungeduldig. Wir haben eine Idee und wollen dann mit dem Kopf durch die Wand, diese dann auch umzusetzen. Dabei gucken wir nicht nach rechts oder links, sondern wollen das einfach machen, manchmal auch unüberlegt. Das ist nicht gut, weil dabei auch viel schief geht. In Deutschland habe ich gelernt, zuerst zu überlegen und nachzudenken, wenn ich eine Idee habe, zu überlegen, wie ich die Idee realistisch umsetzen kann. Das kann manchmal dauern, aber ich habe gelernt, dieses Ziel mit Geduld zu erreichen und das auch was nicht so schnell klappt, aber immer wieder versuchen. Das ist gerade auch wichtig, wenn mehrere Leute bei der Umsetzung eines Projekts beteiligt sind. Dann kann das dauern, aber es ist wichtig alle einzubeziehen und das Projekt nach und nach zu realisieren. Zum Beispiel habe ich die Idee mit den Scholars des KAAD und die Einbeziehung in das Caritas-Projekt. Wenn es nach mir ginge, hätten wir damit schon längst anfangen können, aber es dauert halt noch ein bisschen, da erst einmal die Scholars die Zeit finden müssen und auch Caritas überlegen muss, wo und wie man die in verschiedenen Projekten in Kolumbien nach ihrer Rückkehr einbeziehen kann. Früher hätte ich mir schon Sorgen gemacht, warum das so lange dauert, aber jetzt weiß und sehe ich viel mehr. Die Dinge brauchen halt manchmal ihre Zeit. Ich muss nur etwas Geduld haben und dann kann man das Projekt Schritt für Schritt realisieren" (Interview mit einem kolumbianischen Alumnus in Bogotá, Kolumbien, 2017).

Ein Erlebnis, das uns eine kolumbianische Alumna geschildert hat, macht ihre neue Arbeitsweise deutlich:

„In Medellin hatte ich während meines Forschungsaufenthalts für die Masterarbeit kolumbianische Lehrer zwischen 25 und 60 Jahre in meiner Fortbildungsgruppe. Die waren alle begeistert von der guten Strukturierung meines Lehrplans. Das habe ich in Deutschland gelernt"(Interview mit einer kolumbianischen Alumna in Deutschland, 2018).

Des Weiteren haben fast 70 Prozent der Befragten „sehr viele“ oder „viele“ Organisationsfähigkeiten in Deutschland erlangt. Knapp über 17 Prozent haben immerhin „etwas“ an Strategie- und Organisationsfähigkeiten erlangt $(\mathrm{n}=378)$. Von den Interviewpartner*innen wurden vor allem Zielstrebigkeit und effektives Planen 
und Organisieren als wichtige erlernte Skills genannt. Ein ghanaischer Stipendiat sagte mit Begeisterung:

"Ich habe gelernt, Dinge zu planen und umzusetzen. In Ghana macht man einfach, was gerade gemacht werden muss, ohne einen langfristigen Plan zu haben. Hier habe ich meinen Promotionsplan aufgestellt, um mir die Zeit besser einzuteilen. Das werde ich jetzt auch mit anderen Projekten oder Aktivitäten in meinem Leben machen. Das hilft mir sehr, um die Pläne auch tatsächlich zu verwirklichen"(Interview mit einem ghanaischen Stipendiaten, Skype, 2017).

Ein anderer Ghanaer:

„Ich habe gelernt meine Zeit gut einzuteilen. Aber das ist schwierig hier in Ghana umzusetzen, weil Menschen hier anders denken. Man ist hier dazu gezwungen Kompromisse zu finden. Zum Beispiel, man will eine Projektbesprechung in zwei Stunden fertig bekommen, aber dann dauert es drei oder vier Stunden, weil Leute zu spät kommen, oder wenn sie kommen, dann die Hälfte vergessen haben, oder sich erst einmal längere Zeit über private Dinge unterhalten müssen. Das nervt mich manchmal. Aber man darf sich deswegen nicht fertig machen, sondern man muss Kompromisse dafür finden. Es ist eine andere Kultur hier" (Interview mit einem ghanaischen Alumnus in Accra, Ghana, 2018).

Eine indonesische Stipendiatin lernte in Deutschland selbstständig zu entscheiden und zu planen:

„In Deutschland war ich zum ersten Mal alleine und musste alles selbst organisieren und musste Dinge alleine entscheiden. Das war neu für mich und musste ich erst einmal lernen. Das ist wichtig. In Indonesien habe ich viel mit der Familie oder Freunden gemacht, wir haben auch zusammen entschieden. In Indonesien ist die Familie wichtig, man macht viel zusammen, am Wochenende oder in den Ferien, und man wohnt als Familie auch zusammen" (Interview mit einer indonesischen Stipendiatin, Skype, 2017).

Eine indonesische Alumna hat in Deutschland gelernt mit beruflichen Herausforderungen und mit Konflikten mit Arbeitskollegen konstruktiv umzugehen:

"I think, the most important thing I learned in Germany was how to address problems. You have to know, Indonesia, we don't name problems, or we don't 
talk directly about a mistake of a colleague to a colleague, because that is seemed to be rude. That is our culture. It is based on more social harmony. But in Germany, if there is a problem between colleagues, you mention that problem directly and you try to solve the problem. So, people name the problem directly, and they are not afraid of starting a conflict, because the problem needs to be solved. I think that is good, and I try to adapt that in my working style too. If there is a problem, I have to name it and try to solve it, to make the whole situation better. This is what I learned in Germany. [...] Traditionally, we don't mention problems in public we have with other people, and we don't want to hurt feelings and the thoughts and ideas of someone. This way of thinking and behavior goes back to our tradition of Javanese ethics of harmony. That means that everyone should have and maintain his or her place in the society, and no one should try to get out of this place in society. This also means that no one should improve or getting a position that is not determined for this one. For this reason, Javanese ethics is good for harmony in the society and to keep people calm and focus on their specific work and position, but it is not good for progress and development, because everyone who wants to improve his position or wants to change the status quo is perceived by other members of the society as someone, who tries to destroy the society. But it is not true, he actually wants to improve the society. So if you come back from Germany, where you learned to mention problems and to attack people verbally in a direct way, and you do it in the same way in Indonesia, people can be offended. So you have to be very smart in addressing personal challenges or problems with other people, so you actually don't offend other people, and that they don't take it personally, and the social harmony is not in danger. But that you still can change the situation in the way you want, and that you solve the problem" (Interview mit einer indonesischen Alumna in Jakarta, Indonesien, 2017).

Ein indonesischer Unternehmer führt seine neue Art der Unternehmensführung ebenfalls auf seine Erfahrung in Deutschland zurück:

"Ich habe in Deutschland gelernt, wie man das Unternehmen systematisch führt und die technischen Kenntnisse dazu inhaltlich anwendet. Auch Präzession und Pünktlichkeit habe ich gelernt, was sehr wichtig ist, um auch mit internationalen Partnern zusammenzuarbeiten und um die Arbeit und die Produkte pünktlich abzuliefern. [...] Ich habe auch direktes Reden gelernt, Dinge und Probleme direkt anzusprechen, auch mit meinen Kollegen und Angestellten" (Interview mit einem indonesischen Alumnus in Pontianak, Indonesien, 2017). 
Ein ghanaischer Alumnus:

"Reliability. That is very important to do business, because your partners have to count on you. In Africa, many people do not show up for meetings, even if these are important business meetings. I learned in Germany that the most important thing is to hold to your word, and to realize the things you agreed on with your partners. This commitment is important to be successful in business" (Interview mit einem ghanaischen Alumnus in Deutschland, Skype, 2017).

Eine andere ghanaische Alumna:

„Verantwortung zu übernehmen. Wenn man nicht zur Arbeit kommt, dann braucht man einen wichtigen Grund. In Ghana ist das häufig nicht der Fall. Die Deutschen nehmen Arbeit ernster, und das findet sie gut so, und hat das auch für sich so übernommen. Ich habe gelernt, wie man sich auf etwas im Leben konzentriert und wie man seine Ziele erreicht, und natürlich wie man ruhig bleibt und weitermacht, wenn etwas nicht gleich funktioniert. Man muss fokussiert bleiben im Leben" (Interview mit einer ghanaischen Alumna in Accra, Ghana, 2018).

Ferner wurden auch andere Fähigkeiten genannt, die die Alumni in Deutschland erworben haben, die sich teilweise mit den oben genannten Fähigkeiten überlappen. Dazu gehören u. a. Erwerb der englischen Sprache, leichterer Umgang mit neuen Technologien, höheres Bewusstsein für Umweltschutz (Stichwort „Mülltrennung“), aber auch eine realistischere Einschätzung der negativen Aspekte in westlichen Ländern wie Deutschland, wie z.B. ein hoher gesellschaftlicher Leistungsdruck oder die oben angesprochene „soziale Kälte“. Alle diese Fähigkeiten führten dazu, dass viele der Alumni selbstbewusster geworden sind und sich ihre „Sicht auf die Welt" teilweise grundsätzlich änderte, worauf wir weiter unten noch einmal genauer eingehen.

Open Access Dieses Kapitel wird unter der Creative Commons Namensnennung 4.0 International Lizenz (http://creativecommons.org/licenses/by/4.0/deed.de) veröffentlicht, welche die Nutzung, Vervielfältigung, Bearbeitung, Verbreitung und Wiedergabe in jeglichem Medium und Format erlaubt, sofern Sie den/die ursprünglichen Autor(en) und die Quelle ordnungsgemäß nennen, einen Link zur Creative Commons Lizenz beifügen und angeben, ob Änderungen vorgenommen wurden.

Die in diesem Kapitel enthaltenen Bilder und sonstiges Drittmaterial unterliegen ebenfalls der genannten Creative Commons Lizenz, sofern sich aus der Abbildungslegende nichts anderes ergibt. Sofern das betreffende Material nicht unter der genannten Creative Commons Lizenz steht und die betreffende Handlung nicht nach gesetzlichen Vorschriften erlaubt ist, ist für die oben aufgeführten Weiterverwendungen des Materials die Einwilligung des jeweiligen Rechteinhabers einzuholen. 